\title{
Wörterbuch der Schweizer Sozialpolitik
}

Jean-Michel Bonvin, Pascal Maeder,

Carlo Knöpfel, Valérie Hugentobler,

Ueli Tecklenburg (Hrsg.)
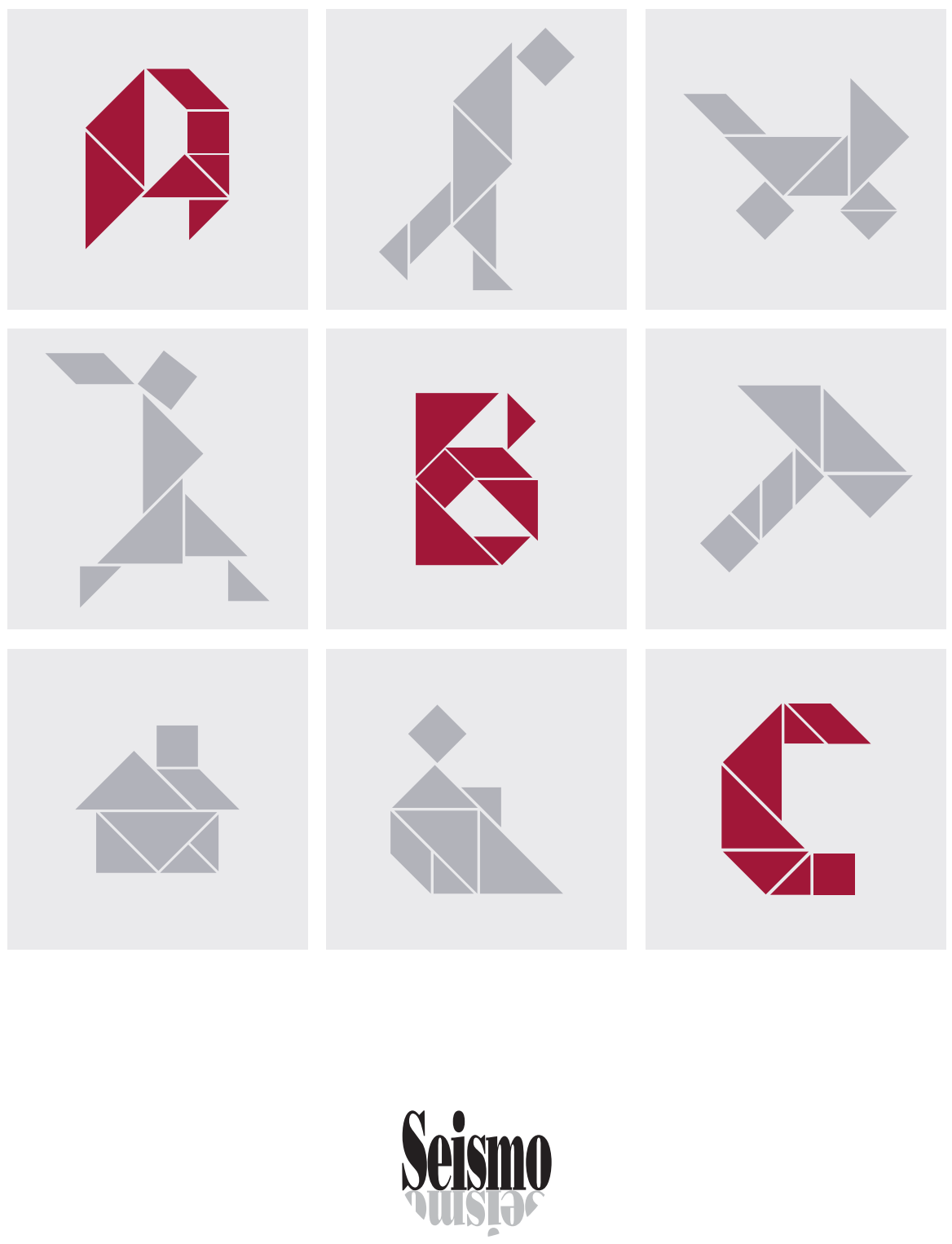
Wörterbuch der Schweizer Sozialpolitik

Jean-Michel Bonvin, Pascal Maeder, Carlo Knöpfel, Valérie Hugentobler und Ueli Tecklenburg (Hrsg.) 



\section{Wörterbuch \\ der Schweizer Sozialpolitik \\ Jean-Michel Bonvin, Pascal Maeder, Carlo Knöpfel, Valérie Hugentobler, \\ Ueli Tecklenburg (Hrsg.)}

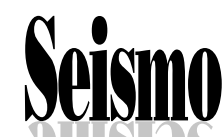


Publiziert mit Unterstützung der folgenden Organisationen: Anonyme Stiftung in Genf; Fachbereich Soziale Arbeit der Fachhochschule Westschweiz; Hochschule für Soziale Arbeit der Fachhochschule Nordwestschweiz; Loterie romande; Nationaler Forschungsschwerpunkt LIVES/ Centre LIVES; Schweizerische Akademie der Geistes- und Sozialwissenschaften; Schweizerische Gemeinnützige Gesellschaft; Schweizerische Vereinigung für Sozialpolitik; Swisslos-Fonds der Kantone Aargau, Basel-Landschaft, Basel-Stadt und Solothurn; VPS Verlag Personalvorsorge und Sozialversicherung. Dem Schweizerischen Nationalfonds zur Förderung der wissenschaftlichen Forschung gebührt Dank für die Unterstützung der Druckvorstufe der französischen Ausgabe dieser Publikation.

Titel der französischen Ausgabe

\section{Dictionnaire de politique sociale suisse}

Publiziert von

Seismo Verlag, Sozialwissenschaften und Gesellschaftsfragen AG

Zürich und Genf

www.seismoverlag.ch

buch@seismoverlag.ch

ISBN 978-3-03777-177-8 (Print)

ISBN 978-3-03777-739-8 (PDF)

https://doi.org/10.33058/seismo.30739

Umschlag: Vincent Freccia, COBRA Communication \& Branding, Lausanne

Dieses Werk ist lizenziert unter einer Creative Commons Namensnennung - nicht kommerziell - keine Bearbeitung 4.0 international Lizenz 
Hinsichtlich der direkten Steuern auf Einkommen und Vermögen natürlicher Personen sowie auf Gewinn und Kapital juristischer Personen sind die Kantone verpflichtet, ihr Steuerrecht in verschiedenen Punkten (steuerpflichtige Subjekte, Berechnungsperioden, Verfahren, Strafbestimmungen usw.) einheitlich zu gestalten. Die Steuertabellen, -sätze und -freibeträge können sie hingegen frei wählen, wobei sie allerdings die Grundrechte gewähren müssen, insbesondere den Grundsatz der Gleichbehandlung und die davon abgeleiteten steuerlichen Prinzipien wie den Grundsatz der Besteuerung nach der Leistungsfähigkeit. Dieser Handlungsspielraum, der auf dem Schweizer Föderalismus beruht, führt zum Steuerwettbewerb zwischen den Kantonen, die sich bemühen, durch besonders günstige Steuersätze oder -tabellen wohlhabende Steuerzahler und zusätzliche Unternehmen anzulocken.

In diesem Zusammenhang haben sich einige Schweizer Kantone für die Flat Rate Tax (Einheitssteuer) entschieden, die anstelle eines progressiven Steuersatzes einen festen Grenzsatz in Verbindung mit einem bedeutenden Steuerabzug vorsieht. Obwohl die meisten Fachleute die Flat Rate Tax als verträglich mit dem Grundsatz der Besteuerung nach der Leistungsfähigkeit einschätzen, weil die Steuerbelastung überproportional zum Einkommensanstieg wächst, wirft dieses System doch Fragen der Steuergerechtigkeit auf. Grund dafür ist, dass diese Besteuerung durch eine «gebremste» Progression gekennzeichnet ist, welche die reichsten Klassen begünstigt und den Grundsatz der Umverteilung des Vermögens untergräbt.

Die Finanzkraft der 26 Kantone unterscheidet sich erheblich aufgrund ihrer geografischen Lage, der ungleichen wirtschaftlichen Entwicklung und weiterer Faktoren, die zu Unterschieden im Steuersubstrat geführt haben. Um diese Diskrepanzen zu verringern und eine gewisse Solidarität zwischen den Kantonen sicherzustellen, wurden Systeme des Finanzausgleichs und des Lastenausgleichs geschaffen. Der Ressourcenausgleich unterscheidet zwischen Kantonen mit hohem und niedrigem Potenzial. Ressourcenschwache Kantone erhalten finanzielle Beiträge von den bessergestellten Kantonen (horizontaler Ausgleich) und vom Bund (vertikaler Ausgleich), über die sie frei verfügen können. Zudem zahlt der Bund Beiträge an die Kantone, die aufgrund ihrer geografischen oder soziodemografischen Besonderheiten höhere Kosten zu tragen haben.

\section{Samuele Vorpe \& Kelly Scapozza}

\section{Literaturhinweise}

Oberson, X. (2012). Droit fiscal suisse (4e éd. entièrement rev. et augm.). Bâle: Helbing Lichtenhahn.

Pedroli, A. (2008). Non solo aliquote: le imposte fra etica, giustizia e concorrenza fiscale. Rivista ticinese di diritto, 1, 11-53.

Yersin, D. (1992). L'égalité de traitement en droit fiscal. Zeitschrift für Schweizerisches Recht, 111(2), 145-297.

\section{Straf- und Massnahmenvollzug}

Aufgabe des Straf- und Massnahmenvollzugs ist der Vollzug freiheitsentziehender Sanktionen mittels verschiedener Vollzugsmodalitäten wie das Festhalten, Inhaftieren und Unterbringen von Personen in öffentlichen oder privaten Institutionen. Die Behörde bestimmt den Bewegungsspielraum von betroffenen Personen. Solche Sanktionen sind seit ca. 200 Jahren für die staatliche Kriminalitätskontrolle zentral. Seit 1942 besteht in der Schweiz ein einheitliches Strafrecht. Der Vollzug der Urteile ist Sache der Kantone, die auch im Rahmen von Strafvollzugskonkordaten die nötigen Anstalten bereitstellen und betreiben müssen.

In der Schweiz gelten folgende Grundsätze für den Freiheitsentzug und beeinflussen die Infrastruktur, das Personal, die Ressourcen und die Vollzugspraxis: 
, Das Prinzip der Rückfallverhütung verlangt eine geeignete Einflussnahme auf die Persönlichkeit und das Verhalten während des Vollzugs mittels der obligatorischen Arbeit, der Bildung, Therapie, Tagesstrukturen und Freizeitgestaltung sowie durch Sozialarbeit begleitete Stabilisierung des künftigen sozialen Umfelds (Familie, soziale Kontakte, Arbeit, Schuldensanierung, Täter-OpferAusgleich, usw.).

> Das Prinzip der Normalisierung verlangt eine weitgehende Angleichung des Alltags in Haft an den Alltag in Freiheit. Dazu gehören alltagskonforme Anforderungen ebenso wie die Nutzung des Vollzugsalltags als Lernfeld für soziales Verhalten (z.B. Gruppenvollzug).

, Das Prinzip der Bekämpfung von Haftschädigung verlangt, Gefangene nicht $\mathrm{zu}$ isolieren und förderliche Sozialkontakte aufrechtzuerhalten (Brief- und Telefonkontakt, Besuche, Sach- und Beziehungsurlaube, Zugang zu Zeitungen, TV, Bücher, in beschränktem Mass auch zum Internet).

> Die Fürsorgepflicht und das Prinzip der Äquivalenz verlangen für den Vollzug ein gleichwertiges Angebot an medizinischer, rechtlicher, sozialer, religiöser und wirtschaftlicher Hilfe wie draussen.

> Die Verhinderung von Straffälligkeit während des Vollzugs sorgt für die Gewährung der inneren Sicherheit zum Schutz von Personal und Mitgefangenen.

Die Vollzugspraxis unterscheidet sich je nach der Sanktion und dem Alter der verurteilten Person. So wird zwischen Strafen und Massnahmen für Erwachsene und Schutzmassnahmen und Strafen für Jugendliche (10.-18. Lebensjahr) unterschieden und klar getrennten Vollzugseinrichtungen zugewiesen. Freiheitsentziehende Strafen werden gemessen am Verschulden und mit einem Enddatum ausgesprochen. (Schutz-)Massnahmen richten sich nicht nach dem Verschulden, sondern nach dem Zweck und den Zielen der Massnahmen, müs- sen überprüft werden und können unterschiedlich oft verlängert werden. Zudem können auch nachträglich, d.h. nach der Strafverbüssung oder in deren Verlauf Massnahmen angeordnet werden. Unterschieden werden therapeutische (etwa in geeigneten Einrichtungen im Zusammenhang mit psychischen Störungen oder Suchtproblematiken) und sicherheitsbezogene Massnahmen, die dem Schutz der Öffentlichkeit dienen. Letztere setzen nach der Verbüssung von Strafen ein, die aufgrund von schweren Taten und bei hoher Wahrscheinlichkeit von Rückfall erfolgen.

Im Straf- auch im Massnahmenvollzug wird nach offenen und geschlossenen Anstaltstypen, mit einem höheren Sicherheitsstandard zur Verhinderung von Flucht oder Ausbruch unterschieden. Die 106 Schweizer Anstalten mit insgesamt 7468 Haftplätzen (Stand 2017) sind im internationalen Vergleich klein bis sehr klein. Nur 4 Anstalten verfügen über mehr als 200 Haftplätze. Etwa die Hälfte der Haftplätze ist für die Untersuchungshaft und etwa zwei Fünftel für den Normalvollzug. Der Rest umfasst den Massnahmenvollzug, die Administrativhaft und alternative Vollzugsformen. Die Belegungsrate lag seit 2003 immer über 80\%. Die kantonale Vollzugsbehörde ist zuständig für die Einweisung und entscheidet in der Regel über Vollzugslockerungen unter Berücksichtigung von Sicherheitsüberlegungen und der Risikoabwägung.

Anstalten sind hierarchisch geführt und umfassen Aufgabenbereiche mit spezialisiertem Personal für die Sicherheit, Betreuung, Arbeit und Bildung, Gesundheits- und Sozialdienst, Verwaltung und Logistik. Wegen der Arbeitspflicht verfügen alle Anstalten des Straf- und Massnahmenvollzugs über Arbeitsateliers. Von zukünftigen Mitarbeitenden wird neben spezifischen fachlichen Anforderungen (Pflegeberufe, Soziale Arbeit, Meisterdiplom in handwerklichen Berufen, usw.) eine abgeschlossene Berufslehre und berufsbegleitende Ausbildung am Schweizerischen Ausbildungs- 
zentrum für das Strafvollzugspersonal in Freiburg verlangt.

Neben der Arbeitspflicht, der Gleichstellung von Arbeit und Bildung sowie therapeutischen Massnahmen ist der Vollzugsplan ein zentrales Instrument des Vollzugs. Dieser wird zwischen der Anstaltsleitung und den Gefangenen ausgearbeitet und periodisch überprüft. Er umschreibt die Vollzugsziele, Unterbringungsart, Beschäftigung, Aus- und Weiterbildung, Therapie, Betreuung und Lockerungsschritte.

Die Arbeitspflicht wird als Grundpfeiler der Wiedereingliederung in die Gesellschaft angesehen auch nach Erreichen des Pensionsalters. Die Arbeit soll möglichst den Fähigkeiten der Person angepasst sein. Das leistungsbasierte Arbeitsentgelt wird teilweise in einem Sperrkonto für die Zeit nach der Entlassung zurückbehalten.

Eine wichtige Rolle spielt die Aufrechterhaltung der Beziehung zur Aussenwelt (Telefon, Lektüre, Briefe, usw.), das Recht auf Besuch und die Möglichkeit, im Rahmen der Progression und der Vorbereitung auf die Entlassung Beziehungs- und Sachurlaube zu gewähren. Wie die Beziehung zur Aussenwelt im Einzelnen gestaltet werden kann, hängt von kantonalen und anstaltsspezifischen Regelungen ab, wobei Entscheide in der Regel durch die zuständige kantonale Vollzugsbehörde gefällt werden.

Der Straf- und Massnahmenvollzug ist mit Herausforderungen konfrontiert, die ihren Ursprung im gesellschaftlichen Wandel der letzten Jahrzehnte haben und eine zunehmende Individualisierung erfordern. Dazu gehören migrationsbedingte Veränderungen der Bevölkerung ebenso wie die zunehmende Alterung der Gesellschaft oder die Prävalenzen psychischer Beeinträchtigungen.

Die Grundhaltung in der Bevölkerung und der Politik beeinflussen zudem die Herausforderung, eine gute Balance von Normalisierung und Progression auf der einen Seite und Aspekten der Sicherheit auf der anderen zu finden. Zurzeit kann eine starke Tendenz zur «Über- sicherung» beobachtet werden, die sich etwa in der restriktiven Entlassungspraxis und der verbreiteten Infragestellung des offenen Strafund Massnahmenvollzugs im öffentlichen und politischen Diskurs äussert. Ein grundsätzliches Problem, das alle staatlichen Massnahmen und Interventionen charakterisiert, ist die Herausforderung, negative Folgen ihrer Segmentierung durch sinnvolle Formen von Übergangsmanagement zu mindern. Das gilt insbesondere im Straf- und Massnahmenvollzug für die Phase der Entlassung und der Begleitung durch die Bewährungshilfe, welche dazu beiträgt, die Investition in den Freiheitsentzug zu sichern und zukünftige, durch erneute Delinquenz verursachte Schäden und Kosten zu verhindern. Die Grundstimmung der Gesellschaft beeinflusst auch deren Bereitschaft, ihre Verantwortung in der Wiedereingliederung von ehemaligen und bestraften Tätern und Täterinnen wahrzunehmen und zu deren Gelingen entsprechend beizutragen. Auch die Anerkennung und Anwendung der verbrieften Grundrechte für Menschen im Freiheitsentzug wird durch die öffentliche Meinung beeinflusst. Es sind zwei Tendenzen erkennbar. Auf der einen Seite steht ein gesteigertes Sicherheitsbedürfnis, das zu einer Fokussierung auf Kontrolle und auch auf das Prinzip des Wegsperrens, welches der Straftheorie der «Unschädlichmachung» entspricht, führt. Auf der anderen Seite sind unter dem Stichwort «Resozialisierung» Bemühungen zu erkennen, den Fokus stärker auf das Schicksal der grossen Mehrheit von Gesetzesübertretenden zu legen, die früher oder später entlassen werden. Die Massnahmen begünstigen die Wiedereingliederung in die Gesellschaft und mindern die Risiken der Rückfälligkeit, damit dem übergeordneten Ziel der Kriminalpolitik, dem Schutz der Allgemeinheit besser entsprochen wird.

\section{Ueli Hostettler}




\section{Literaturhinweise}

Baechtold, A., Weber, J. \& Hostettler, U. (2016). Strafvollzug: Straf- und Massnahmenvollzug an Erwachsenen in der Schweiz (3., vollst. überarb. und erw. Auflage). Bern: Stämpfli.

Brägger, B. F. (Hrsg.) (2014). Das schweizerische Vollzugslexikon: Von der vorläufigen Festnahme zur bedingten Entlassung. Basel: Helbing Lichtenhahn.

Bundesamt für Statistik. Thema «Kriminalität und Strafrecht». https://www.bfs.admin.ch/bfs/de/ home/statistiken/kriminalitaet-strafrecht.html

\section{Strafrechtliche Sanktionen*}

Das Strafrechtssystem, wie wir es heute kennen, basiert auf der Idee, dass die strafrechtliche Sanktion ein geeignetes Mittel zur Bekämpfung der Kriminalität ist. Die strafrechtliche Sanktion stellt dabei das Ergebnis eines gerichtlichen Verfahrens dar, das der Mensch als gesellschaftliche Reaktion auf unzulängliches und als Verbrechen ausgegrenztes Verhalten entwickelt hat. Die Ziele strafrechtlicher Sanktionen lassen sich in zwei grosse Kategorien einteilen, nämlich in moralische und utilitaristische Ziele. Zu den moralischen Zielen gehören die Sühne (die Läuterung der Seele, die durch die Begehung einer Straftat befleckt wurde) und die Vergeltung (die rächende Strafe für verwerfliches Verhalten). Diese moralischen Funktionen bringen keinerlei sozialen Nutzen. In Erwägung, dass jede strafrechtliche Sanktion für die Gesellschaft vorteilhaft sein und/oder durch ihre Ausführung eine «Lektion erteilen» sollte, werden zusätzlich utilitaristische Ziele verfolgt. Dazu gehören die Aufrechterhaltung des sozialen Zusammenhalts und des Kollektivbewusstseins (ein Durkheimscher Begriff, der sich auf die Machtausübung des Staats über die Individuen mittels Sanktionen bezieht), die Resozialisierung (die soziale Wiedereingliederung nach Massnahmen zur Verbesserung der sozialen Fähigkeiten des Individuums), die Einschüchterung (durch die abschreckende
Wirkung der Sanktion auf die schuldige Person, damit sie nicht rückfällig wird, aber auch auf alle potenziell straffälligen Personen - das heisst auf uns alle - durch Einflössen einer gewissen «Angst vor der Polizei»), die Eliminierung (durch die Hinrichtung, Verbannung oder sehr lange Haft), die Wiedergutmachung (durch die Aufforderung oder den Zwang zur Wiedergutmachung des verursachten Schadens) und die Versöhnung zwischen Opfer und Täterschaft (durch einen Prozess der Versöhnung oder Mediation, der eher zur sogenannten restaurative Justiz gehört als zur bei uns vorherrschenden vergeltenden Justiz).

Die strafrechtlichen Sanktionen lassen sich in zwei Kategorien einteilen: einerseits die Strafen, andererseits die Massnahmen. Erstere sollen eine in der Vergangenheit begangene Handlung «bestrafen» und sind daher in der Regel in ihrem Umfang begrenzt, während Letztere von unbestimmter Dauer sind, da sie der besonderen Betreuung eines straffälligen Individuums dienen und daher so lange fortgeführt werden wie nötig. Reicht das Urteil (insbesondere seine einschüchternde und resozialisierende Wirkung) nicht aus, um die Gefahr abzuwenden, dass eine Person erneut eine schwere Straftat begeht, und benötigt die Person eine Behandlung oder stellt sie ein zu grosses Risiko für die Gesellschaft dar, so verhängt das Gericht zusätzlich zur Strafe eine stationäre therapeutische Massnahme oder eine Verwahrung. Im Gegensatz zu einem weitverbreiteten Irrtum haben diese Massnahmen keinerlei strafenden Charakter, da sie sich nicht auf die Vergangenheit der Person und die von ihr begangene Straftat, sondern auf die Zukunft sowohl der Person als auch der Gesellschaft beziehen.

Hinsichtlich der Strafen gibt es in der Schweiz seit dem 1. Januar 2018 Geldstrafen (bemessen in Tagessätzen, deren Anzahl der Schwere des Verschuldens entspricht, während ihre Höhe von der finanziellen Situation der bestraften Person abhängt), Freiheitsstrafen (deren 